\title{
A Survey on Applications of Multi Layer Perceptron Neural Networks in DOA Estimation for Smart Antennas
}

\author{
Subhash Mishra \\ Research Scholar \\ Department of ECE \\ MANIT, Bhopal, INDIA
}

\author{
R. N. Yadav \\ Professor \\ Department of ECE \\ MANIT, Bhopal, INDIA
}

\author{
R. P. Singh \\ Professor \\ Department of ECE \\ MANIT, Bhopal, INDIA
}

\begin{abstract}
Smart antennas use antenna arrays with signal processing algorithms for identifying signals spatially. The direction of arrival (DOA) estimation has a significant role in the implementation of a smart antenna. The conventional and super resolution methods have been used for DOA estimation applications for a long time. These methods have been quite efficient. However, some of them have computational complexities associated with them. Besides, these methods exhibit limited performance in dealing with the correlated signals. Such reasons limit their suitability for real time applications. Neural networks are non linear and use simple mathematical operators. They map the non linear behavior of smart antennas and perform DOA estimations accurately with considerable time reduction. The multi layer perceptron (MLP) based neural networks have demonstrated their capability of estimating DOAs very effectively even for correlated signals.
\end{abstract}

\section{General Terms}

Spatial covariance matrix, Eigen value decomposition, Digital signal processing, Inverse Mapping.

\section{Keywords}

Smart antenna, Neural network (NN), Direction of Arrival (DOA), Multi layer perceptron (MLP).

\section{INTRODUCTION}

The smart antenna technology has emerged as a solution to address various issues related with the wireless networks and therefore, it is one of the most significant areas of study in wireless communications today. Smart antennas utilize spatial diversity aspects like space division multiple access (SDMA) and employ powerful signal processing which enable them to offer many advantages such as increased antenna gain and wider coverage range, reduction of interference and minimization of fading. Hence they are able to provide improvements in the overall capacity of the network and better spectrum utilization [1-2][5-6].The choice of configuration of a smart antenna depends on the application. While the switched beam method can be considered as an extension of cell sectoring, the adaptive arrays have the ability to steer multiple beams towards the desired users and nulls towards the interfering signals [3-4].

Smart antenna techniques are expected to provide efficient use of the spectrum, reduce cost of establishing networks optimize quality of service and support operational transparency in the future wireless networks [7].The concepts of spatial filtering and SDMA are significant in the functioning of smart antennas [5][8]. Detailed illustrations on various technical aspects related with the concepts and functioning of adaptive arrays and smart antennas are presented in [5][6] and [9]. The direction of arrival
(DOA) estimation and beam formation are the key functions of smart antennas. Various DOA algorithms and beam forming techniques related with antenna arrays are described in [1][10]. Conventional algorithms for signal processing find difficulty in handling the non linear behavior of antenna array elements. Super resolution algorithms have shown their effectiveness in estimating DOAs specially for uncorrelated signals even in presence of additive noise but they involve cumbersome computations and are therefore not suitable for use with real time signals [11][14][17] Some methods though quite accurate require highly calibrated antennas [12].

Neural network [18] methods operate with simple mathematical tools and are therefore faster than the traditional superresolution methods. Neural networks have attained a widespread growth over last few decades [19].Their adaptive learning capability and the ability to perform in real time makes them suitable for applications in wireless networks [13][24].The application of neural networks for DOA estimation is based on the inverse of the mapping that antenna array performs. Neural networks like the Hopfield NN, Multi layer perceptron (MLP) $\mathrm{NN}$, Radial basis function (RBF) NN, Hybrid NN and others have been used to perform the task of DOA estimation for smart antennas with accuracy and speed.

Section 2 includes the mathematical analysis of the DOA estimation problem for a uniform linear antenna array. The limitations of the conventional super resolution methods and the applicability of neural networks in DOA estimation are also covered in this section. Section 3 presents the configuration and the principle of working of a multi layer perceptron (MLP) neural network. A summary of various MLPNN based applications for estimation of DOA is covered in Section 4. Section 5 concludes the paper.

\section{ESTIMATION OF DIRECTION OF ARRIVAL (DOA)}

DOA estimation is a very important issue in wireless communications. A number of algorithms for DOA estimation have been developed [1]. They are classified as the classical, maximum likelihood (ML) and the subspace based methods like MUSIC and ESPIRIT [44]. In this section the limitations of the conventional and super resolution techniques are discussed. The mathematical treatment related with the DOA estimation problem for a uniform linear array has been mentioned. The capabilities of the neural networks to solve this also discussed ahead.

\subsection{The Conventional and Super resolution Methods for DOA Estimation}

Several methods have been proposed for DOA estimation of signals in wireless communication systems. The most widely used algorithms like MUSIC (Multiple Signal Classification), 
ESPRIT (Estimation of Signal Parameters via Rotational Invariance Technique), MLE (Maximum Likelihood Estimation) [10] have provided very good results. However, there are some issues related with them which need to be addressed. The MUSIC algorithm is quite efficient in DOA estimation even with additive noise but has computational complexity and has problems in estimating DOA with precision when the data size is small and requires many signal snapshots. ESPRIT has been found to be very efficient and has shown advantages over MUSIC. The abilities of these methods to handle with correlated signals are limited. The MLE method is very accurate and can handle partially correlated signals but it is computationally intensive [32][37]. The matrix pencil (MP) method needs a single snapshot thus requires less computational time [40].

\subsection{Mathematical Analysis}

The mathematical analysis related with the DOA problem for a linear antenna array presented in [1][6][10][16-17][29] is mentioned here. A linear antenna array with $M$ elements and inter space distance $d$ is considered to be exposed to $K(K<M)$ narrow band plane waves impinging on it from directions $\left\{\theta_{l,}, \theta_{2}, \ldots . \theta_{k}\right\}$. The received signal vector of the $i^{\text {th }}$ array element for a linear antenna array is expressed as

$$
X_{i}(t)=\sum_{m=1}^{K} S_{m}(t) e^{-j(i-1) k_{m}}+n_{i}(t)
$$

for $i=1,2,3,4 \ldots \ldots m$. Here $S_{m}(t)$ is the signal of the $m^{\text {th }}$ wave, $n_{i}(t)$ is the noise signal at the $i^{\text {th }}$ sensor and $k_{m}=\frac{\omega_{0} d}{c} \sin \left(\theta_{m}\right)$

Here $c$ is the speed of light. The output of the array in vector notation form can be expressed as

$X(t)=A S(t)+N(t)$

$X(t), S(t)$ and $N(t)$ are given by

$$
\begin{aligned}
& X(t)=\left[\mathrm{X}_{1}(t), \mathrm{X}_{2}(t), \mathrm{X}_{3}(t), \mathrm{X}_{4}(t), \ldots \ldots \ldots \mathrm{X}_{\mathrm{M}}(t)\right]^{\mathrm{T}} \\
& S(t)=\left[\mathrm{S}_{1}(t), \mathrm{S}_{2}(t), \mathrm{S}_{3}(t), \mathrm{S}_{4}(t), \ldots \ldots \ldots \ldots \mathrm{S}_{\mathrm{M}}(t)\right]^{\mathrm{T}} \\
& N(t)=\left[\mathrm{N}_{1}(t), \mathrm{N}_{2}(t), \mathrm{N}_{3}(t), \mathrm{N}_{4}(t), \ldots \ldots \ldots \mathrm{N}_{\mathrm{M}}(t)\right]^{\mathrm{T}} \\
& A=\left[a\left(\theta_{1}\right), a\left(\theta_{2}\right) \ldots a\left(\theta_{m}\right) \ldots a\left(\theta_{k}\right)\right] \\
& a\left(\theta_{m}\right)=\left[1, e^{-j k_{m}}, e^{-j 2 k_{m}}, e^{-j 3 k_{m}}, \ldots \ldots e^{-j(M-1) k_{m}}\right]
\end{aligned}
$$

Here ${ }^{\mathrm{T}}$ denotes the transpose, $A$ is $M \times K$ steering matrix. The spatial correlation matrix $R$ of the array output is defined as

$R=E\left\{X(t) X^{H}(t)\right\}$

$R=A E\left\{S(t) S^{H}(t)\right\}+E\left\{N(t) N^{H}(t)\right\}$

Here, ${ }^{\mathrm{H}}$ is the conjugate transpose. The mapping of antenna array [6][16-17] is considered as $\mathrm{G}: \mathrm{R}^{\mathrm{K}} \rightarrow \mathrm{C}^{\mathrm{M}}$ i.e. from the space of DOAs, $\left\{\Theta=\left[\begin{array}{llllll}\theta_{1} & \theta_{2} & \theta_{3} & \theta_{4} \ldots \theta_{\mathrm{k}}\end{array}\right]^{\mathrm{K}}\right\}$ to the space of array output $X(t)$. The neural network based DOA estimation is based on the inverse mapping i.e. $\mathrm{F}: \mathrm{C}^{\mathrm{M}} \rightarrow \mathrm{R}^{\mathrm{K}}$.

\subsection{Neural Network (NN) Techniques for DOA Estimation}

The concept and development of artificial neural networks (ANN) is motivated from the concept of biological neurons of human brain. Detailed descriptions related with the concept, structure, training and working of neural networks are covered in [18][21].

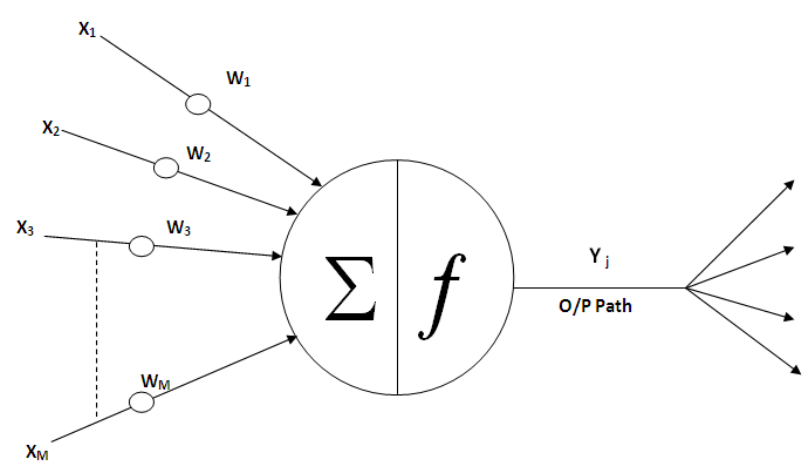

Figure 1. A Basic Neuron Element

A typical basic neuron element is shown in Figure1 [31].The inputs are represented as $X_{i}$, weights are denoted as $W_{i}$. Symbol $\Sigma$ represents 'sum' or 'linear combination' while $f$ represents the 'activation function'. The output path is shown as $\mathrm{Y}_{\mathrm{i} .}$. Neural networks provide simple general purpose mathematical tools to perform complex operations in real time. They are non linear in nature and can handle the behavior of the antenna elements properly. After training and validation they are able to predict the output for the unseen inputs quite accurately. They have enormous parallel processing potential and the ability to provide fast solutions. These capabilities make them suitable for applications in digital communications and wireless networks [23-24][25].

Neural networks present simple data processing models and have the ability to perform in real time even when the number of interferers is more than the antenna elements [15][26]. The neural network techniques are based on the mapping between the spatial correlation matrix $R$ and the angles of arrival [14][16-17]. The input data is the information of correlation matrix while the output layer provides the information about the DOAs. The number of neurons in the input layer of the network depends on the $R$ matrix. The complex matrix of $R$ is required to be normalized before applying to the input layer to reduce the dimension of input vectors for efficient training of NNs [14][4344]. Neural networks such as the Hopfield neural networks, multi layer perceptron (MLP) neural networks, radial basis function (RBF) neural networks and hybrid neural networks have been employed for a variety of applications related with wireless communications. Some reviews on neural networks and their applications in digital communications and smart antennas arrays are available in [22][25][27][28].

\section{MULTI LAYER PERCEPTRON (MLP)}

A multilayer perceptron (MLP) is a multilayered, nonlinear neural network. It consists of multiple layers which are the input layer, one or more hidden layers and an output layer. The activation function of the each hidden layer neuron is nonlinear such as sigmoid or tansigmoid. Each neuron is characterized by the activation function and is connected to all the neurons of the next layer [18][21][23]. Each connection is characterized by its weight factor or synaptic weight. A typical configuration of multilayer perceptron based neural network is shown in Figure 2 . The number of layers in the structure is L. The first layer is the input layer and the $\mathrm{L}^{\text {th }}$ is the output layer. The hidden layers are from the second layer to the $(\mathrm{L}-1)^{\text {th }}$. As seen here the network is fully interconnected allowing each neuron of a layer 
is to be connected to each neuron of the next layer [18][21][23]. This makes forward transmission from the input layer to the output layer through the hidden layers possible. The input signals here can move gradually forward through network. The output of the $L^{\text {th }}$ layer can be expressed as

$$
Y_{l}=F\left(W_{l} Y_{l-1}+B_{l}\right)
$$

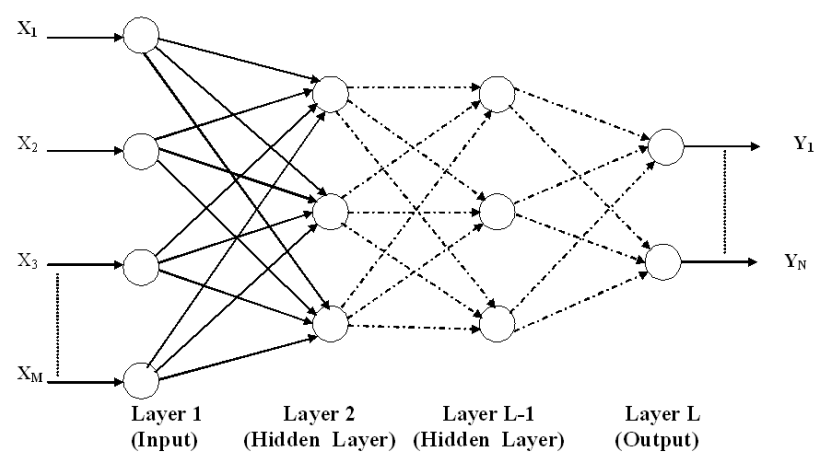

Figure 2. A Typical MLP- NN Configuration

where $F$ is the activation function of each neuron. $Y_{L}$ and $Y_{L^{-1}}$ are outputs of the $L^{\text {th }}$ and $(L-1)^{\text {th }}$ layers, $W_{L}$ is a weight matrix between $(L-1)^{\text {th }}$ and $L{ }^{\text {th }}$ layer and $B_{L}$ is the bias matrix [43][46]. The error signals originating at the output can move layer wise backwards in the network. The process of training is continued till the errors reach to a minimum desired level. The most common training procedure for the MLP is the back propagation (BP) algorithm or its modifications such as Levenberg-Marquardt (LM) or quasi-Newton algorithms. This algorithm takes more memory than conventional back propagation (BP) but is quicker [26][33].

\section{MLPNN BASED DOA ESTIMATION}

Multi layer perceptrons [18-19] have been successfully used in a variety of applications related with wireless communications because of their simple implementation, accuracy and flexibility. A number of studies have presented MLP based neural solutions for DOA estimation of antenna arrays discussed in the foregoing paragraphs. A summary of some of these applications proposed for various antenna arrays during last two decades is given in Table1.

An analysis on the NN based approach for high resolution DOA estimation has been presented in [29]. In this study authors have compared the NN method with MUSIC algorithm and have mentioned about their earlier related work. Signals from two coherent sources at $6^{0}$ and $17^{0}$ are considered to arrive on a 4element uniform linear array (ULA) having element interval of $\lambda / 2$ in this study. The capability of neural networks to handle the direction finding problems for non coherent as well as coherent sources has been shown. It is seen that NN method has clear advantages over MUSIC algorithm when the sources are correlated. Efficient NN based approaches for DOA estimation have been proposed by authors in [30][32]. The precision and speed of the neural networks make them suitable for such applications. In [32] authors have used co-operation of classical MLP and CSW (Constrained Structured Weights) perceptrons for AOA estimations. The results of the method have been comparable to MLE in error minimization besides the method being quick. The method outperforms both MUSIC and
ESPIRIT in conditions when signals are correlated thus showing better performance.

Authors in [31] have proposed a MLP based DOA estimation method. The MLP used here is trained by the back propagation algorithm. The number of hidden layer neurons is 4 . The method is initially applied for a 4-element array, however, shows better performance for an L-shaped antenna array as the later can produce more uniform angular sectors. In [33], a neural beam former using a multiple layer perceptron (MLP) has been presented. A 3-element array is considered here. The MLP used in the study contains 5 elements in the first layer with activation function being tanh and a layer of 4 adaline elements for a linear weighted sum. The number of hidden layer neurons is 5. A set of 36 points (step of $10^{\circ}$ ) is used for training the neural network with LM algorithm. The NN based approach is found to perform better than classical mono pulse system for DOA estimation even in the presence of noise or interference.

A hybrid architecture using RF combining network along with NN having has been described in [34]. The neural network uses combination of MLP and Adalines which perform the classification of DOAs. Two parallel linked MLPs have been used for faster convergence. Each MLP section has three neuron layers. The first layer has 10 perceptrons with tanh activation function. The second layer of 8 adalines and the output layer having 5 perceptrons with sigmoid activation functions. Training of the MLPs is accomplished with the LM algorithm. It is seen that the unexpected performance of the RF hardware can be addressed by the flexibility of the training process making it be suitable for tracking applications.

Authors in [35] have mentioned about the need for taking the non ideal behavior of signals combiners and antenna elements into account. In this study two 3-element linear orthogonal antenna arrays have been used. The system used here is divided into two stages. The signal detection stage with 16 inputs uses an MLP having 10 neurons in the hidden layer. The estimation stage includes two MLPs, each having three layers, the hidden layer with tan sigmoid function and the output layer having linear function. The training algorithm used here is LM. The output (DOA) has to be in the range $45^{\circ}$ to $135^{\circ}$. The errors found are within $2^{\circ}$ for DOA within the $90^{\circ}$ sector of interest.

In [36], the authors have compared the performance of MLP trained with genetic algorithm (GA) to the performance of RBF neural network. The genetic algorithm (GA) helps the MLP to avoid getting stuck in local minima and achieve results with a smaller hidden layer (20 neurons in the hidden layer) as compared to the RBF in the study. In [37], use of ant colony optimization (ACO) method to train MLP having 5 neurons in the hidden layer has been used for DOA estimation. In both cases results have shown the effectiveness of these methods.

Authors in [38] have presented a neural network based DOA estimation method for a switched beam system (SBS). The SBS considered here is an 8-element linear array fed by a Butler Matrix. The method proposed in the study uses multi layer perceptrons (MLPs) having an input layer of 8 nodes fed by the power vectors $P_{m}$ and the output layer provides the angles $\theta_{m}$. The activation function for the hidden layers is hyperbolic tangent and for the output layer it is linear. The training method used in the method is LM so as to achieve faster convergence. 
Table 1 Applications of Multi Layer Percptron (MLP) based Neural Networks for DOA Estimation

\begin{tabular}{|c|c|c|c|}
\hline S.No. & DOA Estimation Application & Type of Antenna Array & Hidden Layer Structure \\
\hline 1 & $\begin{array}{l}\text { Antenna Beamforming for EW Using Adaptive } \\
\text { Layered Networks [31] }\end{array}$ & $\begin{array}{l}\text { 4-element uniform linear } \\
\text { array, } \\
\text { L-shaped array }\end{array}$ & Single hidden layer, 4 neurons \\
\hline 2 & $\begin{array}{l}\text { Co-operation of Multi layer Perceptrons for Angle } \\
\text { of Arrival Estimation [32] }\end{array}$ & 5 sensors & MLP-10 neurons, CSW-2 neurons \\
\hline 3 & $\begin{array}{l}\text { Sectorial Direction Finding Antenna Array with a } \\
\text { MLP Beamformer [33] }\end{array}$ & 3-element antenna array & $\begin{array}{l}5 \text { neurons and } 4 \text { adalines } \\
\text { respectively in the two layers }\end{array}$ \\
\hline 4 & $\begin{array}{l}\text { An Implementation of Direction-Finding Antenna for } \\
\text { Mobile Communications Using a Neural Network } \\
{[34]}\end{array}$ & $\begin{array}{l}\text { Array of } 3 \text { horizontal printed } \\
\text { circular patches }\end{array}$ & $\begin{array}{l}10 \text { perceptrons and } 8 \text { adalines } \\
\text { respectively in the two layers }\end{array}$ \\
\hline 5 & $\begin{array}{l}\text { Implementation of an MLP-based DOA System } \\
\text { Using a Reduced Number of MM-wave Antenna } \\
\text { Elements [35] }\end{array}$ & $\begin{array}{l}\text { Two 3-elements orthogonal } \\
\text { linear arrays }\end{array}$ & $\begin{array}{l}\text { Detection Stage: } 10 \text { neurons, } \\
\text { Estimation Stage: } 30 \text { and } 45 \\
\text { neurons respectively in the two } \\
\text { layers }\end{array}$ \\
\hline 6 & $\begin{array}{l}\text { Performance of Neural Network Trained with } \\
\text { Genetic Algorithm for Direction of Arrival } \\
\text { Estimation [36] }\end{array}$ & 6-element antenna array & $\begin{array}{l}\text { RBF Model: } 42 \text { neurons, } \\
\text { MLP with GA Model: } 20 \text { neurons }\end{array}$ \\
\hline 7 & $\begin{array}{l}\text { A Hybrid Approach Involving Artificial Neural } \\
\text { Network and Ant Colony Optimization for Direction } \\
\text { of Arrival Estimation [37] }\end{array}$ & 5-element antenna array & Single \\
\hline 8 & $\begin{array}{l}\text { Direction of Arrival (DOA) Estimation for a } \\
\text { Switched-Beam DS-CDMA System Using Neural } \\
\text { Networks [40] }\end{array}$ & $\begin{array}{lll}\text { 8-element } & \text { uniform } & \text { linear } \\
\text { array fed by } 8 \times 8 & \text { Butler } \\
\text { Matrix } & & \\
\end{array}$ & $\begin{array}{l}\text { Two hidden layers, Hyperbolic } \\
\text { tangent }\end{array}$ \\
\hline 9 & $\begin{array}{l}\text { On the DOA Estimation for a Switched Beam } \\
\text { Antenna System Using Neural Networks [41] }\end{array}$ & $\begin{array}{l}\text { 8-element uniform linear } \\
\text { array (micro strip patches) } \\
\text { fed by } 8 \text { x8 Butler Matrix }\end{array}$ & $\begin{array}{l}\text { Uniform Illumination.: } 8 \text { and } 24 \\
\text { neurons in the two layers } \\
\text { respectively, Cosine Illumination. : } \\
7 \text { and } 14 \text { neurons in the two layers }\end{array}$ \\
\hline 10 & $\begin{array}{l}\text { mpact Neural Network Based } \\
\mathrm{m}[42]\end{array}$ & $\begin{array}{l}\text { Two orthogonal 2-element } \\
\text { patch arrays }\end{array}$ & $\begin{array}{l}\text { 2D NNDE : } 20 \text { neurons, 2D } \\
\text { NNDC: } 8 \text { neurons }\end{array}$ \\
\hline 11 & $\begin{array}{l}\text { Neural Network Based Direction of Arrival } \\
\text { Estimation for a MIMO OFDM Radar [43] }\end{array}$ & $4 \times 4$ & $\begin{array}{l}16 \text { and } 20 \text { neurons respectively in } \\
\text { the two layers }\end{array}$ \\
\hline 12 & $\begin{array}{l}\text { Application of Neural Networks in Spatial Signal } \\
\text { Processing [44] }\end{array}$ & $\begin{array}{l}\text { Rectangular antenna array } \\
\text { with } 16 \text { elements for } 2 \mathrm{D} \\
\text { DOA estimation case }\end{array}$ & $\begin{array}{l}\text { Single MLP : } 18 \text { neurons each in } \\
\text { the two layers, } \\
\text { TSS MLPs : Detection- } 12,10 \\
\text { neurons in two layers Estimation- } \\
12,12 \text { neurons each in the two } \\
\text { layers }\end{array}$ \\
\hline 13 & $\begin{array}{l}\text { Application of Artificial Neural Networks for } \\
\text { Efficient High-Resolution 2D DOA Estimation [45] }\end{array}$ & $\begin{array}{l}\text { Uniform rectangular array } \\
\text { with16 elements }\end{array}$ & $\begin{array}{l}\text { RBF Model : } 219 \text { neurons, } \\
\text { MLP+RBF Model : } 22 \text { neurons } \\
\text { each in the two layers of MLP, } 81 \\
\text { neurons in RBF }\end{array}$ \\
\hline 14 & $\begin{array}{l}\text { Efficient Neural Network Approach for } 2 \mathrm{D} \text { DOA } \\
\text { Estimation Based on Antenna Array Measurements } \\
{[46]}\end{array}$ & $\begin{array}{l}4 \times 4 \text { rectangular micro strip } \\
\text { patch array }\end{array}$ & $\begin{array}{l}\text { MLP (Azimuth) }: 20 \text { and } 12 \\
\text { neurons in the two layers, MLP } \\
\text { (Elevation) : } 16 \text { and } 12 \text { neurons in } \\
\text { the two layers respectively. }\end{array}$ \\
\hline
\end{tabular}

In this method only power measurements at a single point of the system are needed resulting in reduction of cost and complexity of hardware. The method is found to provide accurate results even for a large number of incoming signals unlike the majority of conventional DOA estimation algorithms. In [39] and [40] this method has been effectively used for DS-CDMA systems. The method shows very good results for large number of simultaneous users. It is shown that for 40 users and processing gain of 128 the root mean square error is within $1^{0}$. This method has been compared with other super resolution techniques and found to be adaptable to the DOA estimation for multipath signals also. Further in [41], the authors have presented the application of methods mentioned in [38-40] for the DOA estimation process considering uniform and cosine illuminations respectively for an 8-element array fed by Butler matrix $(8 \times 8)$. A data set of approximately 5000 pairs of $P_{m}$ and $\theta_{m}$ is used for training. The training algorithm is LM. The performance of the method presented is independent of the signal amplitudes and the total number of mobile users. For uniform illumination the input layer has 8 nodes. The two hidden layers have 8 and 24 nodes respectively. For cosine illumination there are 7 nodes in the input and the two hidden layers have 7 and 14 nodes respectively. Authors have also proposed modifications to perform estimation of DOA with variations in power levels. Cosine illumination with sector beam has been considered for this. The MLP configuration for this case employs 7 nodes in input layer while the hidden layers have 14 and 28 nodes respectively. The use of sector beam has been found to improve the performance. Almost $95 \%$ of the estimations have been found with error of $0.5^{\circ}$. Besides, it is also seen that DOA can still be estimated quite accurately with root mean square error less than $1^{\circ}$ even when the signal is 6 
dB down from the interferers. A compact DOA system based on two orthogonal 2- element patch arrays has been proposed in [42]. The neural network processes the normalized amplitudes of three beams to determine the DOA. In this study the MLPs are used for NNDE (for DOA estimation) and NNDC (for DOA classification).The input nodes of both NNDE and NNDC have 6 neurons each. The hidden layer for NNDE has 20 perceptrons and for NNDC the hidden layer has 8 perceptrons. The output layer of NNDE has 2 linear neurons while NNDC requires only 1 (single) neuron with a sigmoid activation function. The error in the DOA varies from $0.8^{0}$ to $1.8^{0}$ for $5^{0}$ to $45^{0}$ range.

Use of a standard multilayer perceptron neural network (MLP$\mathrm{NN}$ ) to determine the angular positions of signals for a $4 \times 4$ MIMO radar has been suggested in [43]. The azimuth angles of the two signals are considered for the range $-20^{\circ}$ to $20^{\circ}$, in steps of $1^{0}$. The authors have suggested use of MLP-NN with 31 neurons in the input layer, 1 neuron in the output layer 16 and 20 neurons in the hidden layers for optimum results. The method outperforms MUSIC in terms of processing time. In [44], authors have proposed applications of different models. Here two different MLP based models have been used for 2D DOA estimation. The first approach uses single MLP based DOA estimation and the other uses a two stage sectorization (TSS-NN) based DOA estimation where two MLPs are used. A 16-element rectangular antenna array is used in the study to collect the data for the training. Results demonstrate the suitability of both the methods. The single MLP based model requires 18 neurons in each of its two hidden layers. The two stage MLN model requires 12 and 10 neurons respectively in the two hidden layers for the detection stage and 12 neurons each in the two hidden layers for the estimation stage.

Another comparison of different approaches can be found in [45]. One approach is based on single RBF neural networks and the other known as sectorization uses a combination of both MLP and RBF neural networks. In the second approach detection of a source signal in a sector is performed using MLP networks and then the DOA is estimated using RBF. A 16element uniform rectangular array (URA) having inter-element spacing of $\lambda / 2$ provides the data set for training. The MLP needs to be trained with less data as it is required to perform classification of input vectors. It is seen that for the single RBF based method optimum performance is achieved with 219 neurons in the hidden layer. In the second method the optimum results are possible with 22 neurons in each of the two hidden layers of MLP used for detection and 81 neurons in the hidden layer of the RBF used for estimation. Both models are more suitable for real-time situations as compared to 2D MUSIC. The RBF+MLP model provides smaller errors in DOA estimation than the single RBF model besides reducing the training time. In [46], authors have presented a neural networkbased model for estimation of 2D DOA. A $4 \times 4$ rectangular micro strip patch has been used along with a horn antenna in a set up. The MLPs employed to measure the azimuth angles use 20 and 12 neurons in the two hidden layers respectively while the MLP for elevation angle has 16 and 12 neurons respectively. The MLPNN model is able to handle the effects arising due to geometrical inaccuracies of the array, the connectors, the cables and the measurement conditions and proves its effectiveness in 2D DOA estimation.

\section{CONCLUSIONS}

The limitations of the conventional methods and super resolution techniques in terms of tedious computations required and limited efficiency in estimating DOA for correlated signals can be resolved by the neural network techniques. Neural networks perform the basic mathematical operations and calculate elementary functions. They are therefore faster as compared to the computationally intensive DOA algorithms. Being non linear in nature they are able to map the antenna parameters effectively. As evident from the variety of studies incorporated in this review, the MLP based neural networks have been excellent in DOA estimations. They have demonstrated flexibility of applications and ability to adapt with different learning algorithm. They may have slow convergence during training (off line) but it does not affect their speed and high accuracy levels in real time situations. MLPNNs are capable of performing with small number of neurons in hidden layers. They can be implemented with simple hardware. MLP based neural networks are robust and efficient. Their performance in case of strongly correlated sources has been found very effective and they present one of the most attractive set of tools for smart antennas applications.

\section{ACKNOWLEDGEMENTS}

We express our sincere thanks to the authors and the publishers of all the books, research papers/articles/reviews referred in this paper.

\section{REFERENCES}

[1] H. Krim and M. Viberg, "Two Decades of Array Signal Processing Research: A Parametric Approach", IEEE Signal Processing Magazine, pp. 67-94, July 1996

[2] J. H. Winters, "Smart Antennas for Wireless Systems", IEEE Personal Communications, vol.5, issue 1, pp.23-27, 1998.

[3] M. Chryssomallis, "Smart Antennas", IEEE Antennas and Propagation Magazine, vol. 42, no.3, pp.129-136, June 2000.

[4] S. Bellofiore, C.A. Balanis, J.Foufz, and A.S. Spanias, "Smart-Antenna Systems for Mobile Communication Networks Part I: Overview and Antenna Design", IEEE Antennas and Propagation Magazine, vol.44, no.3, 2002.

[5] L. C. Godara, "Smart Antennas", Boca Raton: CRC Press LLC, 2004.

[6] A. H. El. Zooghby, "Smart Antenna Engineering", Artech House, 2005.

[7] A. Alexiou and M. Haardt, 'Smart Antenna Technologies for Future Wireless Systems: Trends and Challenges', IEEE Communications Magazine, pp. 90-97, Sept 2004.

[8] R. H. Roy, "An Overview of Smart Antenna Technology: The Next Wave in Wireless Communications", IEEE Aerospace Conference, vol.3, pp.339-345, 1998.

[9] Lal C. Godra, “Applications of Antenna Arrays to Mobile Communications Part1: Performance Improvement, Feasibility, and System Considerations" Proceedings of IEEE vol. 85, no.7 pp. 1031-1060, July 1997.

[10] Lal C. Godra, “Applications of Antenna Arrays to Mobile Communications, Part 2: Beamforming and Direction of Arrival Considerations", Proceedings of the IEEE, vol. 85, no.8, pp. 1195-1245, Aug.1997.

[11] Titus K. Y. Lo, Henry Leung, and John Litwa, "Artificial Neural Network for AOA Estimation in Multipath Environment over the Sea", IEEE Journal of Oceanic Engineering, vol. 19, issue 4, pp. 555-562, Oct.1994 
[12] H. L. Southhall, J. A. Simmeres, and T. H. O’Donnell, "Direction Finding in Phased Arrays with a Neural Network Beamformer", IEEE Transactions on Antennas and Propagation,vol.43, no.12, Dec.1995

[13] S. Bang, S. H. Sheu, and J. Bing, "Neural Network for Detection of Signals in Communications", IEEE Transactions Circuits and Systems I: Fundamental Theory and Applications, vol. 43, no. 8, pp. 644-655, Aug. 1996.

[14] A. H. El. Zooghby, C. G. Christodoulou, and M Georgiopoulos, "Performance of Radial Basis Function Networks for Direction of Arrival Estimation with Antenna Arrays", IEEE Transactions on Antennas and Propagation, vol. 45, no. 11, pp. 1611-1617, Nov. 1997.

[15] Y.S. Hwu and M. D. Srinath, "A Neural Network Approach to Design of Smart Antennas for Wireless Communication Systems", Record of $31^{s t}$ Asilomar Conference on Signals, Systems and Computers, vol.1, pp.145-148, Nov. 1997.

[16] A. H. El Zooghby, C. G. Christodoulou, and M. Georgiopoulos, "A neural network based smart antenna for multiple source tracking”, IEEE Transactions on Antennas and Propagation, vol. 48, pp.768-776, May 2000.

[17] M. Sarevska, B. Milovanovic, and Z. Stankovic, "Alternative Signal Detection for Neural Network Based Smart Antenna", Proceedings of $7^{\text {th }}$ International Seminar on Neural Network Applications in Elect. Engineering, NEUREL, pp.85-89, Sept. 2004.

[18] S. Haykins, "Neural Networks : A Comprehensive Foundation”, IEEE Press, New York, 1994.

[19] B. Widrow , M. A. Lehr, "A 30 Years of Adaptive Neural Networks: Perceptron, Madaline and Backpropagation" Proceedings of the IEEE, vol.78, issue 9, pp. 1415-1442, 1990.

[20] Kar-Ann Toh and Chong-Yee Lee, "Efficient Network Training For DOA Estimation", Systems, Man, and Cybernetics, IEEE SMC '99 Conference Proceedings, vol. 5, pp. 383-388, 1999.

[21] Q. J. Zhang and K. C. Gupta, "Neural networks for RF and Microwave Design", Artech House, July 2000.

[22] K.L. Du, A. K. Y. Lai , K. K. M. Cheng, and M. N. S. Swamy, "Neural Methods for Antenna Array Signal Processing: A Review", Elsevier Science B. V., Signal Processing, vol. 82, issue 4, pp 547-561, Apr. 2002.

[23] Q. J. Zhang, K. C. Gupta and V. K. Devabhaktuni, "Artificial Neural Networks for RF and Microwave Design-From Theory to Practice", IEEE Transactions on Microwave Theory and Techniques, vol. 51, No. 4, pp.1339-1350, April 2003.

[24] A. Patnaik, D. E. Anagnostou, R. K. Mishra, C. G Cristodoulou, and J. C. Lyke, "Applications of Neural Networks in Wireless Communications", IEEE Antennas and Propagation Magazine, vol. 46, no.3, pp. 130-137, June 2004.

[25] M.Ibnkahla, “Applications of Neural Networks to Digital Communications - A Survey" Elsevier Signal Processing Journal, vol. 80, issue 7, pp.1185-1215, July 2000.

[26] L. Merad, F. T. Bendimerad, S. M. Meriah, and S. A. Djennas, "Neural Networks for Synthesis and
Optimization of Antenna Arrays", Radio Engineering, vol. 16, no.1, pp. 23-30, Apr.2007.

[27] S. Mishra, R. N. Yadav and. R. P. Singh, "Neural Networks in Smart Antenna Design for Co-Channel Interference (CCI) Reduction: A Review", Proceedings of ICWMC 2009, pp. 228-233, Oct.2009.

[28] A. Rawat, R. N. Yadav, and S. C. Shrivastava, "Neural Network Applications in Smart Antenna Arrays: A Review", International Journal of Electronics and Communication (AË̈ Elsevier), vol. 66, pp. 903-912, Nov. 2012.

[29] Y. M. Wang and Y. L. Ma, "The Performance of Neural Network for High Resolution Direction of Arrival Estimation", Proceedings of International Conference on Circuits and Systems, pp. 301-304, June 1991.

[30] G. Burel \& N. Rondel, "Neural Networks for Array Processing: From DOA Estimation to Blind Separation of Sources", International Conference on Systems, Man and Cybernetics, vol.2, pp. 601-606, Oct.1993.

[31] P.C.J. Hill and P.D. Wells, "Antenna Beamforming for EW Using Adaptive Layered Networks", IEE Colloquium, London, pp. 2/1-2/5 Jan 1994.

[32] N. D. M. Rondel and G Y Burel, "Co-operation of Multi layer Perceptrons for Angle of Arrival Estimation", Proceedings of $3^{\text {rd }}$ International Conference on Document Analysis and Recognition, vol.2,pp. 1141-1144,1995.

[33] E. Charpentier and J.J. Laurin, "Sectorial Direction Finding Antenna Array with a MLP Beamformer", IEEE Antenna and Propagation Society International Symposium, vol. 4, pp. 2270-2273, July 1997.

[34] E. Charpentier and J.J. Laurin, "An Implementation of a Direction Finding Antenna for Mobile Communications Using a Neural Network", IEEE Transactions on Antennas and Propagation, vol. 47, pp. 1152-1159, 1999.

[35] E. Danneville, J.J. Brault, and J.J. Laurin, "Implementation of an MLP-based DOA System Using a Reduced Number of MM-wave Antenna Elements", Proceedings of International Joint Conference on Neural Networks, Montreal, Canada, pp 3220-3225, 2005.

[36] H. M. Pour, Z. Atlasbaf, A. Mirzaee and M.Hakak, "Performance of Neural Network Trained with Genetic Algorithm for Direction of Arrival Estimation", Proceedings of First Mobile Computing and Wireless Communications International Conference, pp.197-202, 2006

[37] H. M. Pour, Z. Atlasbaf, A. Mirzaee, and M. Hakak, “A Hybrid Approach Involving Artificial Neural Network and Ant Colony Optimization for Direction of Arrival Estimation", Canadian Conference on Electrical and Computer Engineering, CCECE, pp.1059-1064, 2008.

[38] K. A. Gotsis, E. G.Vaitsopoulos, K. Siakavara, and J. N. Sahalos, "Multiple Signal Direction of Arrival (DOA) Estimation for a Switched-Beam System Using Neural Networks", Proceedings Progress in Electromagn. Research Symposium, Prague, Czech Republic, pp.420424, Aug 2007.

[39] K. Gotsis, T. Kaifas, K. Siakavara, and J. Sahalos, "Direction of Arrival (DOA) Estimation for a SwitchedBeam DS-CDMA System Using Neural Networks", 
Proceedings of $19^{\text {th }}$ International Conference Appl. Electromagn. Communications, Croatia, pp. 47-50, Sept 2007.

[40] K. A. Gotsis, T. N. Kaifas, K. Siakavara, and J. N. Sahalos, "Direction of Arrival (DOA) Estimation for a Switched-Beam DS-CDMA System Using Neural Networks", J. Automatika, vol. 49, no. 1-2, pp. 27-33, 2008 .

[41] K. A. Gotsis, K. Siakavara, and J. N. Sahalos, "On the Direction of Arrival (DOA) Estimation for a Switched Beam Antenna System Using Neural Networks", IEEE Transactions on Antennas and Propagation, vol. 57, No.5, pp.1399-1411, May 2009.

[42] N. J. G. Fonseca, M. Coudyser, J. J. Laurin, and J. J. Brault, "On the Design of a Compact Neural NetworkBased DOA Estimation System", IEEE Transactions on Antennas and Propagation, vol. 58, No. 2, February 2010.

[43] Y. L. Sit, M. Agatonovic, and T. Zwick, "Neural Network Based Direction of Arrival Estimation for a MIMO OFDM
Radar", Proceedings of the $9^{\text {th }}$ European Radar Conference, EuRAD, pp.298-301, Oct-Nov 2012.

[44] B Milovanovic, M. Agatonovic, Z. Stankovic, N. Doncov, and M. Sarevska, "Application of Neural Networks in Spatial Signal Processing", $11^{\text {th }}$ Symposium on Neural Network Applications in Elect. Engineering, NEUREL2012, Serbia, 2012.

[45] M. Agatonovic, Z. Stankovic, N. Doncov, L. Sit , B Milovanovic, and T. Zwick, "Application of Artificial Neural Networks for Efficient High-Resolution 2D DOA Estimation", Radioengineering, vol.21, no.4, pp. 11781186, Dec 2012.

[46] M. Agatonovic, Z. Stankovic, I. Milovanovic, N. Doncov, L. Sit , B Milovanovic, and T. Zwick, "Efficient Neural Network Approach For 2D DOA Estimation Based On Antenna Array Measurements", Progress in Electromagnetics Research, vol. 137, pp.741-758, 2013. 